\title{
Optical design for efficient light emission in OLEDs and anisotropic layers
}

\author{
Lieven Penninck ${ }^{\mathrm{a}, \mathrm{b}}$, Kristiaan Neyts*a,b \\ ${ }^{a}$ ELIS Department, Ghent University, Sint-Pietersnieuwstraat 41, B-9000 Gent, Belgium; ${ }^{b}$ Center for \\ Nano and Biophotonics, Ghent University, Sint-Pietersnieuwstraat 41, B-9000 Gent, Belgium.
}

\begin{abstract}
The light emission from an electrical dipole antenna is determined by the orientation of the antenna and the optical properties of the materials that surround it. For the outcoupling efficiency of an OLED it is beneficial if the dipole moment of the luminescent transition is parallel with the substrate. In some OLEDs a preferential orientation of the emitting molecules can indeed be observed. In this paper we discuss how anisotropy of the dipole orientation and optical anisotropy of the materials influence the light emitted from a planar structure. Numerical simulations are verified with measurements of OLEDs (for the dipole orientation) and dye doped liquid crystals (for the optical anisotropy).
\end{abstract}

Keywords: OLEDs, anisotropy, dipole orientation, outcoupling, dipole emission

\section{INTRODUCTION}

Traditionally it is assumed that OLEDs consist of a stack of isotropic materials: metal cathode, ITO anode, hole transport layer (HTL), electron transport layer (ETL) and emitting layer. There are indications that some layers in the OLED stack are not isotropic and this anisotropy can have important benefits.

The most important benefit is connected with the anisotropy of the emitting molecules. The dipole transition from an exciton to the ground state is determined by the spatial distribution of the wave functions and thus by the orientation of the emitting molecule in space. An electrical dipole antenna does not emit light in the direction parallel to the axis and as a result, a dipole transition with axis perpendicular to the substrate will couple less light into air than a dipole transition with axis parallel to the substrate. Recently emission pattern ${ }^{1}$ and decay rate ${ }^{2}$ measurements have provided evidence for the fact that the dipole emitters in an OLED can indeed be oriented. The origin of the anisotropy of the emitter molecules can be in the deposition geometry: it can be expected that planar (or elongated) molecules that are evaporated onto a planar substrate will bind more strongly when both planes (or the plane and the long axis) are at a smaller angle. So if the molecules have some rotational freedom after reaching the substrate they will tend to orient parallel to the substrate. A strong anisotropy in the emission does not necessarily mean that the optical properties (permittivity tensor) is strongly anisotropic, because the concentration of the (oriented) emitting molecules in the EML is usually small. However, a small optical anisotropy can be expected.

The motivation for having an anisotropy in the OLED materials may also be in the electrical functionality. In order to increase the conductivity of the ETL or the HTL in a desired direction, anisotropic materials may be used. It is known that disc-shaped organic molecules with columnar stacking may have a strongly increased conductivity in the direction of the column axis ${ }^{3}$. The spontaneous ordered stacking of organic molecules can occur by passing through a nematic liquid crystalline phase, in which the molecular interaction and the corresponding free energy cause the organic molecules to align.

Liquid crystalline materials have optical properties which are usually strongly anisotropic. The dielectric anisotropy is used to switch the liquid crystal director along the applied electric field and the optical anisotropy is used to change the polarization state of the light in a liquid crystal display. Light emission from oriented liquid crystalline phases is relevant for a number of applications. One application is liquid crystal lasers, in which a dye in a chiral nematic liquid crystal emits light along the helical axis of the chiral nematic phase ${ }^{4-7}$. The alignment of the dye along the local director (and parallel to the helical axis) improves the efficiency of the emission and reduces the threshold for lasing.

*kneyts@elis.ugent.be; phone +32 92643381 ; lcp.elis.ugent.be

Organic Light Emitting Materials and Devices XVII, edited by Franky So, Chihaya Adachi, Proc. of SPIE

Vol. $8829,88291 \mathrm{H} \cdot 02013 \mathrm{SPIE} \cdot \mathrm{CCC}$ code: $0277-786 \mathrm{X} / 13 / \$ 18 \cdot \mathrm{doi}: 10.1117 / 12.2027197$ 
In this paper we describe how the anisotropy of a material influences the emission of light. The first approach is to consider the orientation of the electrical dipole transition in an otherwise isotropic material. The orientation has an impact on the angle dependency of the emission and on the decay rate. In a second step we consider the emission from an optically anisotropic medium, which involves using an anisotropic permittivity tensor for the dipole emission simulations. As many OLEDs and light emitting liquid crystals have a one-dimensional structure, we limit ourselves to planar stacks containing isotropic and anisotropic media.

\section{ORIENTED DIPOLE EMITTERS IN AN ISOTROPIC MEDIUM}

\subsection{Dipole emission}

The theory for the emission of a dipole antenna in an isotropic thin film stack is well known. The emission can be calculated by decomposing the radiation from an elementary electrical dipole antenna into plane (and evanescent) waves and by adding all contributions from transmitted, reflected and partially reflected plane waves coherently ${ }^{8}$. In this description, the total emission is an integral over $\kappa$, the periodicity of plane (in this case it is equal to the projection of the wave vector $k$ ) or evanescent waves on the $x y$-plane of the substrate. The dipole radiation has many contributions: a part of it is emitted into air $\left(\kappa<k_{\text {air }}\right)$, a part reaches the substrate $\left(\kappa<k_{\text {substrate }}\right)$, a part is absorbed in the organic layer (when the organic layers are absorbing) and a part is absorbed in the metallic cathode. It turns out that the part that is absorbed by the metallic electrode is very different for parallel and perpendicular dipoles ${ }^{9}$.
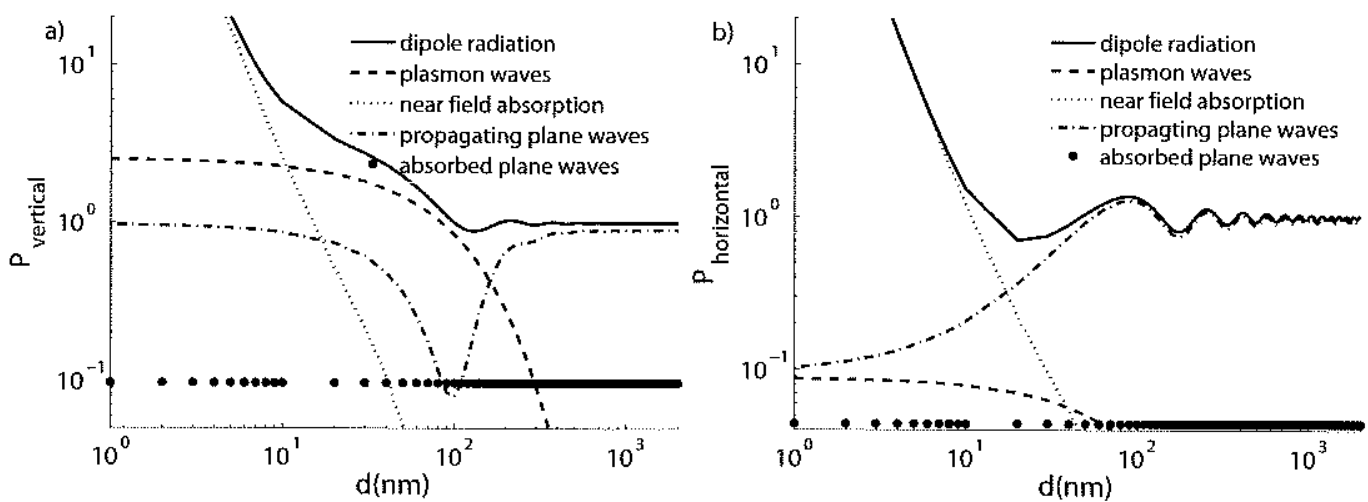

Figure 1. Simulated contributions in the emitted power for perpendicular (left) and parallel (right) dipole emitters as a function of the distance from the mirror. Solid line: total power based on the plane wave decomposition. Dashed line: power based on the analytical formula for plasmon coupling. Point line: power based on the analytical formula for near-field emission. Point-dashed line: plane waves emitted in the medium $n_{\mathrm{a}}$. Dots: power of the plane waves $\left(\kappa<\mathrm{k}_{\text {organic }}\right)$ that is absorbed in the mirror.

The diagrams in Figure 1 compare the different contributions for a dipole emitter in a perfectly transparent dielectric medium as a function of the distance $d$ from the metallic mirror. The parameters used in the simulations are: $\lambda=530 \mathrm{~nm}$ for the wavelength (which is the maximum of the emission spectrum of $\mathrm{AlQ}_{3}$ ); $n_{\mathrm{a}}=1.70$ for the refractive index for the organic layer $\left(\mathrm{AlQ}_{3}\right)$ and $n_{m}=\varepsilon+j \sigma=0.88+j 6.45$ for the complex refractive index for the metal (aluminium $^{10}$ ). The contributions of plasmon waves can be estimated from analytical formulas:

$$
\begin{aligned}
& P_{\text {vertical }}^{\text {plasmon }}=\frac{3 \pi \sigma^{6} n_{a} \exp \left(-2\left|k_{z, a}^{\prime}\right| d\right)}{\left(\sigma^{4}-n_{a}^{4}\right)\left(\sigma^{2}-n_{a}^{2}\right)^{3 / 2}} \\
& P_{\text {horizontal }}^{\text {plasmon }}=\frac{3 \pi \sigma^{4} n_{a}^{3} \exp \left(-2\left|k_{z, a}^{\prime}\right| d\right)}{2\left(\sigma^{4}-n_{a}^{4}\right)\left(\sigma^{2}-n_{a}^{2}\right)^{3 / 2}}
\end{aligned}
$$


with $k_{z, a}^{\prime}=j \frac{k_{0} n_{a}^{2}}{\sqrt{\sigma^{2}-n_{a}^{2}}}$ the z-component of the plasmon wave vector. Note that the contribution for the vertical dipole is considerably higher, because $\sigma^{2} n_{a}^{-2}>>1$. In this analytical approximation the real part of the refractive index is neglected. The contributions of near-field emission can also be estimated from analytical formulas:

$$
\begin{aligned}
& P_{\text {vertical }}^{\text {near-field }}=\frac{3}{2} \frac{\sigma \varepsilon}{n_{a} k_{0}^{3}\left(-\sigma^{2}+n_{a}^{2}\right)^{2}} \frac{1}{d^{3}} \\
& P_{\text {horizontal }}^{\text {near-field }}=\frac{3}{4} \frac{\sigma \varepsilon}{n_{a} k_{0}^{3}\left(-\sigma^{2}+n_{a}^{2}\right)^{2}} \frac{1}{d^{3}} .
\end{aligned}
$$

For this example, the near-field power is only important for very small distances from the metallic mirror. It is striking that the plasmonic contribution is much smaller for the parallel dipole than for the perpendicular dipole. This is because the plasmonic mode has an electric field which is mainly perpendicular to the surface of the metallic layer.

\subsection{Outcoupling efficiency}

From the previous section it is clear that only a part of the light is emitted and not absorbed in the electrode. The part that is emitted into air (or into the substrate) is the useful part, so we can define an efficiency for outcoupling into air (or into the substrate) as the fraction of the total power of the dipole antenna emitted into air (or into the substrate). It is well known that the distance between the dipoles and the metallic mirror determines if the directly emitted and the reflected light interfere constructively ${ }^{11}$. We will therefore simulate the efficiency for a device with structure given in Figure $2 \mathrm{c}$, for varying ETL thickness, first assuming that the dipole emitters are randomly oriented. Figure $2 \mathrm{a}$ gives the power (referenced to the power of a elementary dipole antenna in a homogeneous medium) emitted by a randomly oriented dipole antenna into different parts: into air, into the substrate (including the contribution into air), into plane waves in the organic layer (including the contributions into air and the substrate) and finally the total power $F$ (including also the absorbed evanescent waves). Note that the total power of the dipole antenna depends on the optical environment. For an exciton, only one photon can be emitted, therefore, it makes sense to scale the contributions in different parts with respect to the total power that is emitted for that ETL thickness. This is illustrated in Figure $2 \mathrm{~b}$.
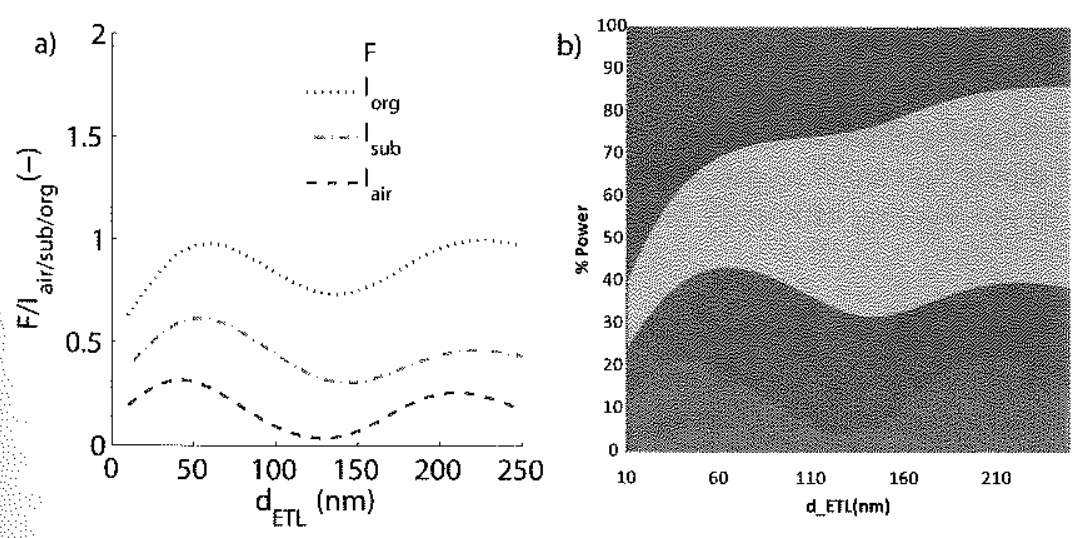

c)

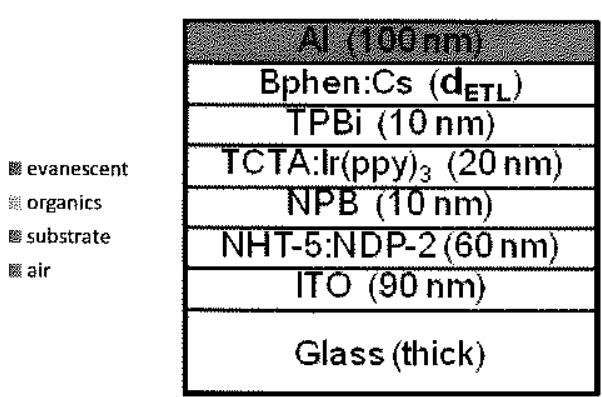

Figure 2. a) Power of a randomly oriented emitter (in air, in the substrate, in the organics, total) as a function of the thickness of the ETL normalized by the power of an emitter in a homogeneous medium. b) Same as in a, but now normalized to the total power for the same thickness of the ETL. c) Test structure for the simulation of the emission power.

\subsection{Dipole orientation and efficiency}

Let us now consider dipole antennas that are oriented parallel or perpendicular to the substrate, or have a distribution that is not randorn. In this section we assume that the distribution does not depend on the azimuthal angle, which is valid for devices which have rotational symmetry around the axis perpendicular to the substrate. We define the parameter $\alpha$, which is the effective fraction of parallel dipoles. For a given density distribution $\rho$ over the solid angle, which is a function of the inclination angle $\theta_{\text {dipole }}$, the factor $\alpha$ is given by: 


$$
\alpha=\int_{\nu=0}^{\pi / 2} \sin ^{3} \theta_{\text {dipole }} \cdot \rho\left(\theta_{\text {dipoole }}\right) \cdot d \theta_{\text {dipose }} .
$$

This fraction is zero for perpendicular dipoles $\left(\theta_{\text {dipole }}=0\right)$, one for parallel dipoles $\left(\theta_{\text {dipole }}=\pi / 2\right)$, and $67 \%$ for randomly oriented dipoles $(\rho=1)$. If we define the normalized total power for parallel and perpendicular dipoles as $F_{/ /}$and $F_{\perp}$ and the normalized emission into air (or the substrate) as $I_{/ /}$and $I_{\perp}$, then the outcoupling efficiency in air (or into the substrate) for an ensemble of dipoles with given distribution $\rho$ and corresponding factor $\alpha$ is given by:

$$
\eta_{\text {out }}(\alpha)=\frac{\alpha I_{/ /}+(1-\alpha) I_{\perp}}{\alpha F_{/ /}+(1-\alpha) F_{\perp}} \text {. }
$$

In Figure $3 b$ (and 3a) we calculate the fraction of the power that is emitted into air (or into the substrate, including the contribution into air) as a function of the ETL thickness, for different values of the parameter $\alpha$, for the same structure as shown in Figure 2c. The parallel dipoles yield the highest outcoupling efficiencies, in air as well as in the substrate. Due to interference effects, there is a strong modulation as a function of the ETL thickness, with the parallel dipole having a maximum in the outcoupling efficiency into air around $40 \mathrm{~nm}$ and $210 \mathrm{~nm}$ and the perpendicular dipole having a (much lower) maximum near $130 \mathrm{~nm}$.
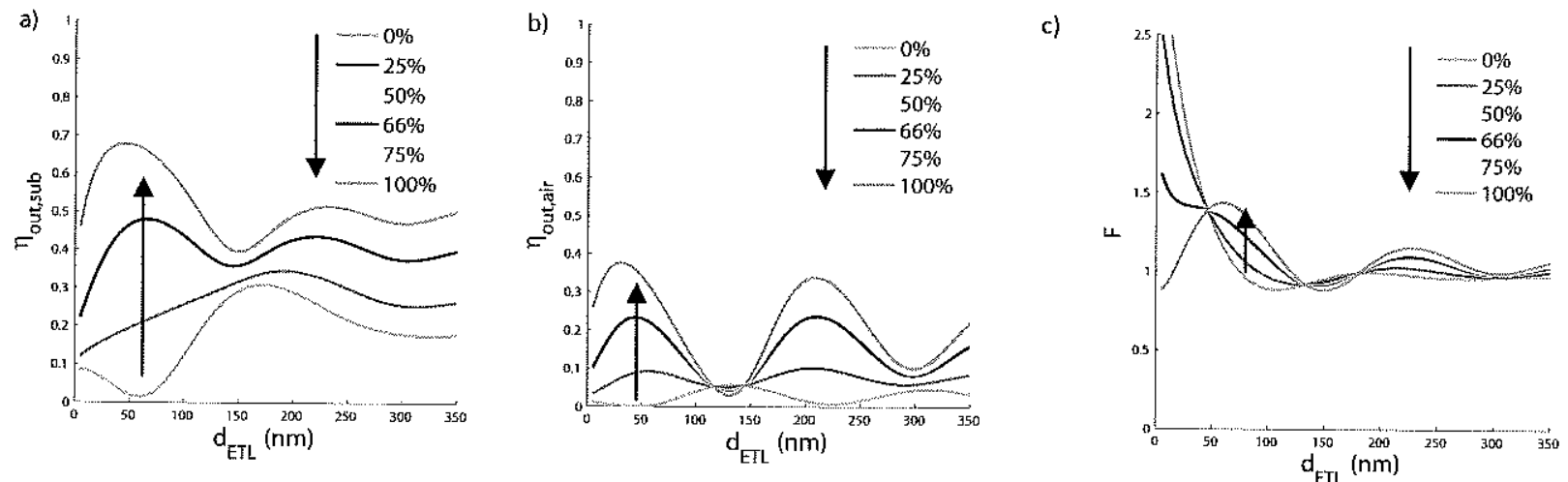

Figure 3. Simulated outcoupling efficiency for a distribution of dipole emitters characterized by the parameter $\alpha$ (the effective fraction of parallel oriented dipole antennas) as a function of the thickness of the ETL layer. a) outcoupling efficiency into the substrate; b) outcoupling efficiency into air; c) total emission $F$ (integrating all contributions, emitted and absorbed).

\subsection{Decay rate of oriented dipole antennas}

It was mentioned previously that parallel and perpendicular dipole antennas have different emission patterns and different total emission power. As only one photon is emitted per exciton, the increase into the power of the dipole antenna translates into an increase of the decay rate (and a decrease of the decay time) for the emission. A variation in the decay rate as a function of the thickness of the ETL layer has indeed been observed ${ }^{2}$. In principle, the decay rate may have contributions from both radiative decay (changing with the optical environment and proportional with the total emitted power $F$ ) and from non-radiative decay (independent of the environment) ${ }^{12}$. However, for the material shown in Figure 4 the contribution of the non-radiative decay is small. 


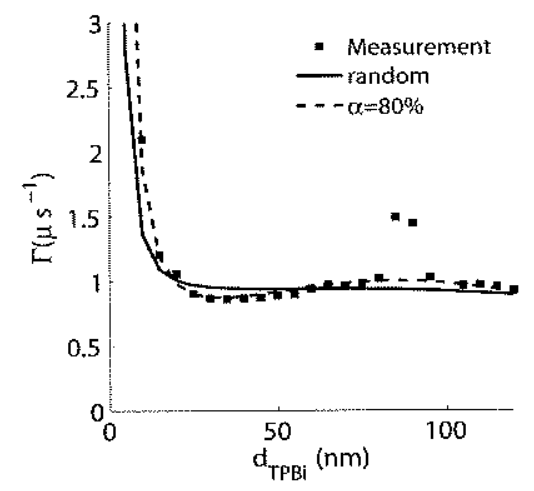

Figure 4. Measured and fitted photoluminescent decay rates for an OLED structure with $\operatorname{Ir}(\mathrm{MDQ}) 2(\mathrm{acac})$. With an effective alignment factor of $\alpha=80 \%$ (dashed line) the measured decay rates are reproduced very well. Random alignment ( $\alpha=67 \%$, solid line) does not give a good fit.

\section{EMISSON FROM ANISOTROPIC MEDIA}

When an elementary dipole is immersed in a homogeneous anisotropic (uniaxial) medium, the radiation pattern depends on the relative orientation of the dipole and the optical axis of the medium. We developed an algorithm to calculate the emission (including the angle and wavelength dependency) from an electrical dipole in a one-dimensional stack of anisotropic layers ${ }^{13}$.

As anisotropic layers in OLEDs are not readily available, we test the theoretical results on other anisotropic structures: dye-doped chiral nematic liquid crystals (LC). E7 (Merck) is a liquid crystal with wide temperature range and strong anisotropy. The refractive indices vary between $n_{0}=1.53$ and $n_{e}=1.75$ at $500 \mathrm{~nm}$ and $n_{0}=1.51$ and $n_{e}=1.72$ at $780 \mathrm{~nm}$. The liquid crystal is doped with a chiral molecule BDH1305 (Merck) to obtain a right handed periodic helix with the director parallel to the glass substrate. Finally, the dye DCM (Exciton) is added to obtain a luminescent mixture that can be excited with a $532 \mathrm{~nm}$ laser ${ }^{14}$. The material has a pitch of $365 \mathrm{~nm}$ and this leads to a band gap for the transmission of right-handed polarized light between $n_{0} P=555 \mathrm{~nm}$ and $n_{\mathrm{e}} P=635 \mathrm{~nm}$.

a)

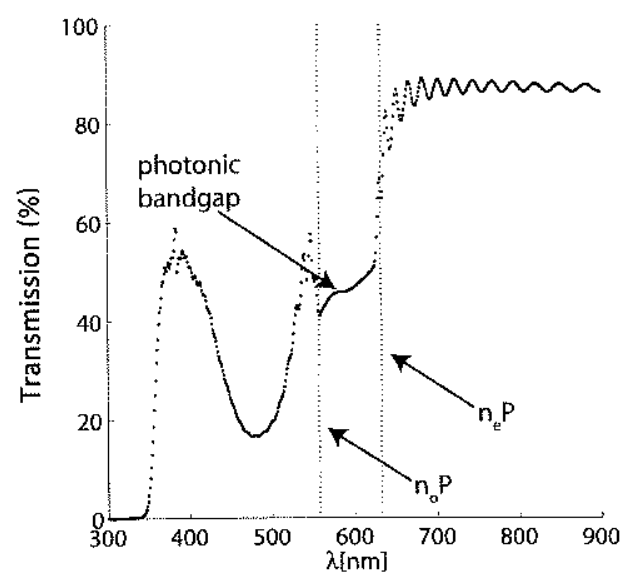

b)

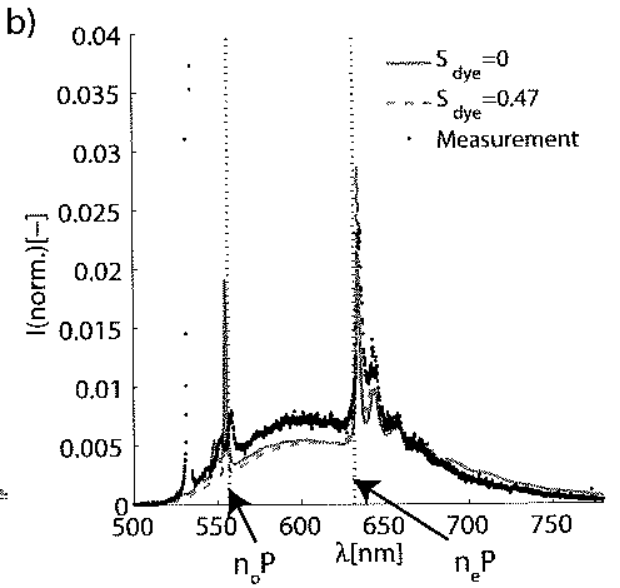

Figure 5. a) Measured transmission spectrum for chiral nematic LC showing the dye absorption around 500nm and the photonic band gap for right handed polarized light. b) Measured and simulated spontaneous emission of the dye DCM in the periodic LC structure.

In the transmission spectrum of the LC (Figure $5 \mathrm{~b}$ ) there is a dip around $500 \mathrm{~nm}$ corresponding to the absorption of the dye DCM, and a dip between $555 \mathrm{~nm}$ and $635 \mathrm{~nm}$ corresponding to the photonic band gap of the periodic structure. The emission spectrum is measured in the direction perpendicular to the substrate. In the band gap there is mainly emission of 
left-handed polarized light, whereas the peaks near the short and long edges of the band gap region emit mainly righthanded polarized light. The measured spectrum shows a peak around $532 \mathrm{~nm}$ which is due to scattered light of the exciting laser. The emission spectrum depends on how well the dye molecules are oriented along the local director of the liquid crystal. The comparison between theory and simulations indicates that the order parameter for the dye molecules is about 0.47 , which means that they are mainly aligned along the director.

\section{CONCLUSION}

The emission of light from an isotropic or anisotropic one-dimensional structure can be simulated by decomposing the dipole radiation into plane waves and taking into account partial reflections. The orientation of the dipole transition in the OLED stack has an important influence on the outcoupling efficiency: dipoles parallel to the substrate are more efficient that dipoles that are perpendicular to the substrate. Experimental evidence for the preferential orientation of $\operatorname{Ir}(\mathrm{MDQ}) 2$ (acac) molecules parallel to the substrate is obtained from decay time measurements. The simulations for light emission from materials with optical anisotropic are verified by measuring the emission from chiral nematic liquid crystal doped with dye molecules. There is very good agreement between the observed and the simulated emission spectra.

\section{ACKNOWLEDGEMENT}

The authors would like to acknowledge the financial support by the Interuniversity Attraction Poles program of the Belgian Science Policy Office (grant IAP P7-35); by the IWT (Flemish Institute for Science and Technology) and by the European Community's Seventh Framework Program (Grant Agreement No. FP7-224122, OLED100.eu)

\section{REFERENCES}

[1] M. Flammich, M. C. Gather, N. Danz et al., "Orientation of emissive dipoles in OLEDs: Quantitative in situ analysis," Organic Electronics 11, 1039-1046 (2010).

[2] L. Penninck, F. Steinbacher, R. Krause et al., "Determining emissive dipole orientation in organic light emitting devices by decay time measurement," Organic Electronics 13, 3079-3084 (2012).

[3] B. R. Kaafarani, "Discotic Liquid Crystals for Opto-Electronic Applications," Chemistry of Materials 23, 378396 (2011).

[4] J. Schmidtke, W. Stille, H. Finkelmann et al., "Laser emission in a dye doped cholesteric polymer network," Advanced Materials 14, 746-+ (2002).

[5] H. Coles, and S. Morris, "Liquid-crystal lasers," Nature Photonics 4, 676-685 (2010).

[6] L. Penninck, J. Beeckman, P. De Visschere et al., "Numerical simulation of stimulated emission and lasing in dye doped cholesteric liquid crystal films," Journal of Applied Physics 113, (2013).

[7] J. Schmidtke, W. Stille, and H. Finkelmann, "Defect mode emission of a dye doped cholesteric polymer network," Physical Review Letters 90, (2003).

[8] K. A. Neyts, "Simulation of light emission from thin-film microcavities," Journal of the Optical Society of America a-Optics Image Science and Vision 15, 962-971 (1998).

[9] L. Penninck, S. Mladenowski, and K. Neyts, "The effects of planar metallic interfaces on the radiation of nearby electrical dipoles," Journal of Optics 12, - (2010).

[10] M. j. Weber, [Handbook of optical materials] CRC Press, (2003).

[11] S. K. So, W. K. Choi, L. M. Leung et al., "Interference effects in bilayer organic light-emitting diodes," Applied Physics Letters 74, 1939-1941 (1999).

[12] S. Mladenovski, S. Reineke, and K. Neyts, "Measurement and simulation of exciton decay times in organic light-emitting devices with different layer structures," Optics Letters 34, 1375-1377 (2009).

[13] L. Penninck, P. De Visschere, J. Beeckman et al, "Simulating the Emission Properties of Luminescent Dyes within One-Dimensional Uniaxial Liquid Crystal Microcavities," Molecular Crystals and Liquid Crystals 560 , 82-92 (2012).

[14] L. Penninck, J. Beeckman, P. De Visschere et al., "Light emission from dye-doped cholesteric liquid crystals at oblique angles: Simulation and experiment," Physical Review E 85, (2012). 